\title{
Derivation and Application of a New Equation for Design and Analysis of Triple Spool Mixed Turbofan Jet Engines with Verification
}

\section{Foroozan Zare, Árpád Veress}

Department of Aeronautics, Naval Architecture and Railway Vehicle

Budapest University of Technology and Economics

Mủegyetem rkp. 3, H-1111 Budapest, Hungary

e-mail: fzare@vrht.bme.hu; averess@ vrht.bme.hu

\begin{abstract}
The development of an improved mathematical model is reported, herein, for modeling the thermo-dynamic processes of triple spool mixed turbofan jet engines with an afterburner, at start conditions with special care for the verification. The T-s diagram and the main characteristics of the engines are determined by a concentrated parameterdistribution type method, implemented in the MATLAB environment. The governing equations are based on mass, energy balance and the real thermo-dynamic processes. $A$ non-linear constraint optimization method is used, for identifying the unknown parameters. Temperature and component mass fraction dependent gas properties are calculated by iteration cycles in case of functional dependencies. A new and more accurate equation is derived and applied for determining the critical pressure at converging nozzle flow with consideration of the local material properties. The thermo-dynamic analyses are completed for NK-32 and NK-25 turbo jet engines. The plausibility of the method and the verification the new equation is provided.
\end{abstract}

Keywords: triple spool turbojet engine, thermodynamics, mathematical model, verification

\section{Nomenclature}

\section{Variables(Latin)}

$A_{9}$

$C_{f}$

$C_{p}$

$\bar{C}_{p}$

$d_{9}$

$D$

$f$
Outlet area of the engine $\left(\mathrm{m}^{2}\right)$

Specific heat of the fuel $(\mathrm{J} / \mathrm{kg} / \mathrm{K})$

Specific heat $(\mathrm{J} / \mathrm{kg} / \mathrm{K})$

Mean (between $T_{i}$ and $\left.T_{i+1}\right)$ specific heat $(\mathrm{J} / \mathrm{kg} / \mathrm{K})$

Outlet diameter of the engine (m)

Inlet diameter of the engine (m)

Fuel to air ratio $(\mathrm{kg} / \mathrm{kg})$ 


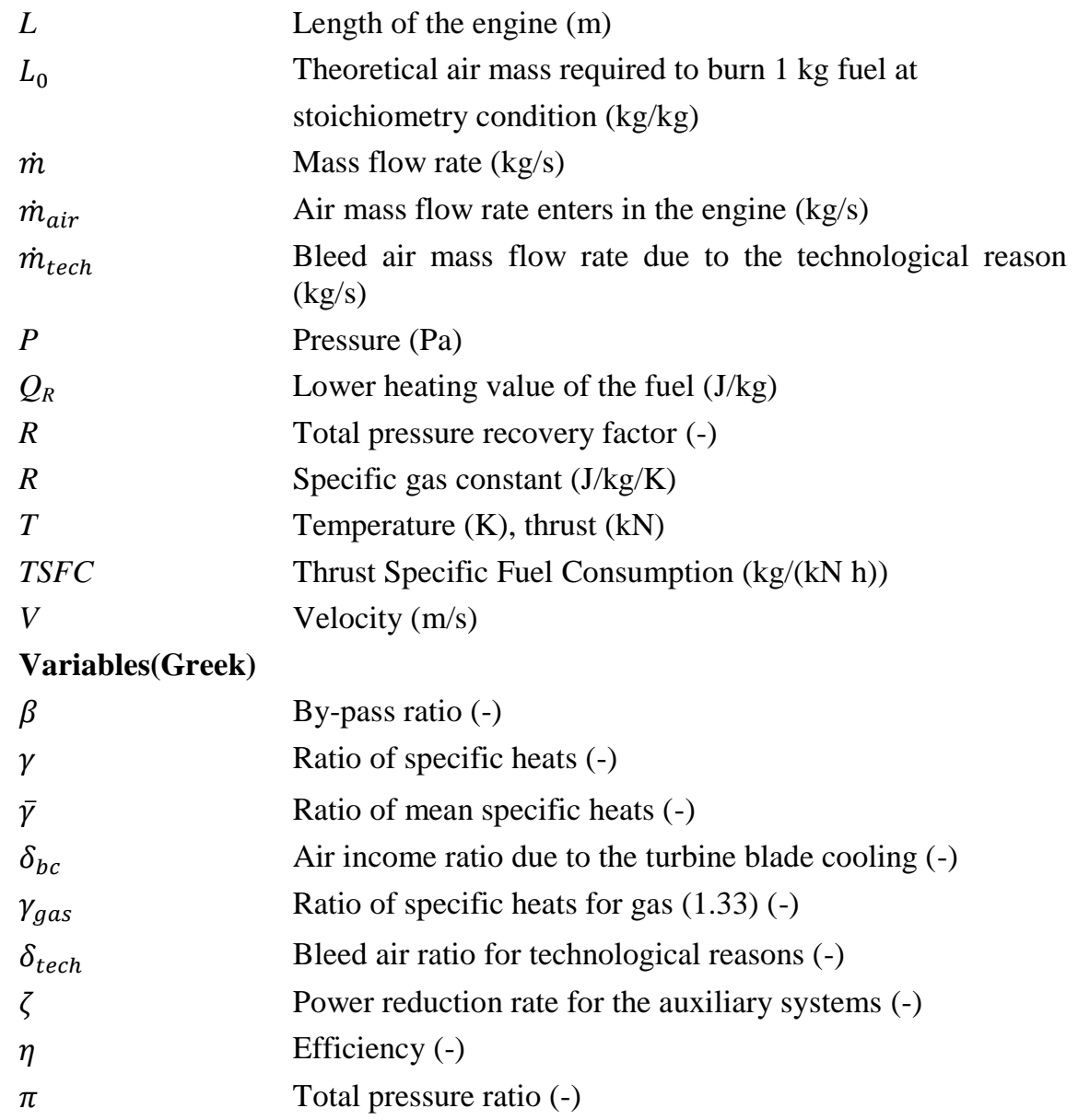

\section{Subscripts}

0

0-9

$1,2,3$

A

A

Al

C

C

Cc

B
Total

Engine cross sections

Number of turbine and compressor spool

Afterburner

Ambient

Afterburner liner

Compressor

Critical

Combustion chamber

Burning 


$\begin{array}{ll}B c & \text { Blade cooling } \\ D & \text { Diffuser } \\ F & \text { Fuel, fan } \\ H p & \text { High pressure } \\ I p & \text { Intermediate pressure } \\ L p & \text { Low pressure } \\ M & \text { Mechanical } \\ m i x & \text { Mass flow weighted parameter for air-gas mixture } \\ N & \text { Nozzle } \\ S & \text { Isentropic } \\ S t & \text { Stoichiometric condition } \\ T & \text { Turbine } \\ t e c h & \text { (Mass flow) re-movement for technological reason }\end{array}$

\section{Introduction}

A significant number of leading technologies are established and transferred within the aeronautical sector. A wide range of $R \& D$ activities are in progress in fields, such as, the application of control theories [1,2], feasibility study of a new system-solution [3], improvement of component characteristics [4], new approach for noise modeling [5] and diagnostics [6].

This is especially true for the propulsion systems of the aircraft, as it is going to be presented in the next paragraphs and chapters. Moreover, beside the aeronautical applications, the gas turbines are generally used in energy generation in the other contributions of transportation; they can be found in the energetics, oil and gas sectors of the industry. These types of engines have higher power-density ratios ( $15-22 \mathrm{~kW} / \mathrm{kg})$ compared to piston engines $(\sim 0.7-1.5 \mathrm{~kW} / \mathrm{kg})$. The gas turbines are relatively light-weight structures and have a compact size, which makes their installation cost efficient. These engines are less sensitive for overloads; they have less solid cross sectional area against the upstream flow (less drag) and have less vibration due to the well balanced and rather axisymmetric rotating components. The gas turbines have high availability factor (80-99\%) and their reliability can be over $99 \%$. They have low emission (there is no lubricant in the combustion chamber and no soot during transient loads) they contain less moving parts and represent less sensitivity for the quality of the fuel used, compared to piston engines. Additionally, there is no need for liquid-based cooling system, but the maximum allowable temperature (e.g. $\sim 1450^{\circ} \mathrm{C}$ ) at the turbine inlet section must be limited due to metallurgical factors. 
Besides, the technical characteristics of the gas turbines today, a certain amount of potential is available for improving their efficiencies, power and emissions. Although the experiences and the know-how of the gas turbine manufacturers increasing continuously, the different mathematical models with using of optimum choice and form of the most dominant processes can significantly contribute to decrease cost, time and capacity in the early phase of gas turbine design and developments. There are many scientific publications are subjected to the thermodynamic-based simulation approaches, which confirms also the need for creating more and more accurate calculation methods. Guha [7] determined the optimum pressure ratio of fan both in analytical and numerical way for separate stream and mixed stream bypass engines. It has been presented that the optimum fan pressure ratio depends on the thrust and it is a weak function of the bypass ratio. Two simple, explicit relations have been derived for determining the optimum fan pressure ratio in bypass engines. The results of the analytical equations are compared with the output of the numerical optimization. Silva and his co-workers [8] shown an evolutionary approach called the StudGA which is an optimization design method. The purpose of his works is to optimize the performance of the gas turbine in terms of minimizing fuel consumption at nominal thrust output, and simultaneously to maximize the thrust of the same fuel consumption as well as to decrease turbine blade temperature.

Recently, three spool mixed turbofan engines are frequently used in commercial and military applications due to their high power density and efficiency and so low normalized range factor and emission at relatively high flight speed and at wide operational range. Hence, the NK-32 (see Figure 1) and NK-25 turbo jet engines are considered for testing and verifying the results of the presently applied mathematical model. The Kuznetsov NK-32 is an afterburning 3-spool low bypass turbofan jet engine, which powers the Tupolev Tu-160 supersonic bomber, and was fitted to the later model Tupolev Tu-144LL supersonic transporter. It is the largest and most powerful engine ever fitted on a combat aircraft. It produces 245 $\mathrm{kN}$ of thrust in maximum afterburner [13]. The Kuznetsov NK-25 is a turbofan aircraft engine used in the Tupolev Tu-22M strategic bomber. It can equal the NK-321 engine as one of the most powerful supersonic engines in service today. It is rated at $245 \mathrm{kN}$ thrust. It was superior to many other engines because of its improved fuel consumption [14].

A concentrated parameter distribution-type method has been developed in the present paper to determine the thermo-dynamic cycles of three spool mixed turbofan engines. Mass and energy balance with real thermo-dynamic processes are used in the computational procedure. The unknown input parameters are determined by a constraint nonlinear optimization. The goal function of the optimization is to minimize the difference between the calculated and available thrust and thrust specific fuel consumption if they are given in the datasheet of the engine. Concerning the material properties, iteration cycles are implemented to evaluate the temperature and component mass fraction dependent gas parameters 
as specific heat at constant pressure and ratio of specific heats. A new equation has been derived for determining the critical pressure at converging nozzle flow with considering real thermodynamic conditions. The verifications of the results are carried out by considering available technical specifications, followed by the parameter identifications for the unknown parameters.

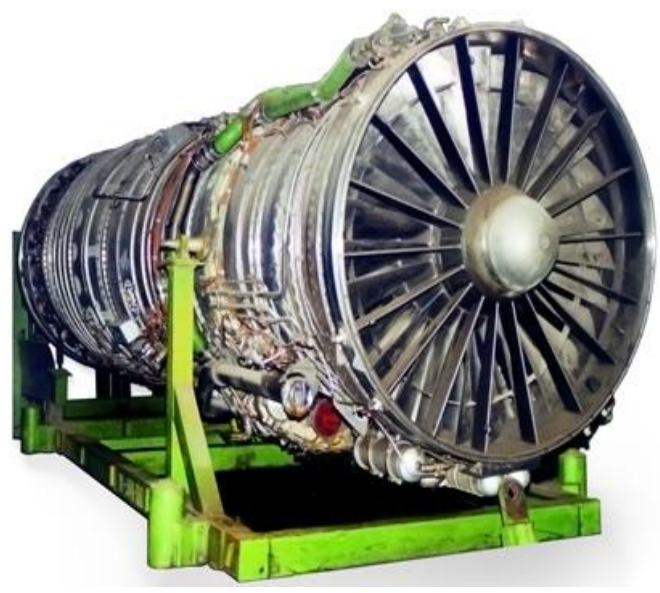

Figure 1

NK-32 turbofan jet engine [10]

\section{Thermo-Dynamic Model for the Triple Spool Turbofan Engine}

Description of the applied engines, assumptions and the modelling approach for the gas turbine has been discussed in the present chapter.

\subsection{Introduction and General Remarks}

A layout with the considered cross sections of a typical triple spool turbojet engine with afterburner is presented in Figure 2. The environmental parameters as pressure and temperature at static sea level conditions belong to section " 0 " are obtained by ISA (International Standard Atmosphere) and valid at start conditions:

- Ambient static pressure:

$$
\begin{aligned}
& p_{a}=101325 \mathrm{~Pa} \\
& T_{a}=288 \mathrm{~K}
\end{aligned}
$$

- Ambient static temperature:

The ambient air enters into the engine at section " 1 ". The operational fluid suffers from pressure drop in the inlet diffuser, which is between port " 1 " and " 2 ". The compressed air is generated from cross section " 2 " to " 3 ". The compressor unit 
consists of three main segments as low, medium and high pressure components. The low pressure compressor unit operates as fan module also and the by-passed air leaves the downstream section of the last fan stage is not directly exhausted, but it flows in a duct around the engine core and it is mixed with the hot gases leaving the turbine at section " 6 ". The combustion chamber is located between port " 3 " and " 4 ", where the heat is generated by adding fuel to the compressed air at stoichiometric condition and at burning activation temperature. The flow stream with high total enthalpy expands and provides energy to the high, medium and low pressure turbines, which is transmitted to the high, medium and low pressure compressor spool respectively. The afterburner for increasing thrust is located section " 6 " and " 7 ". The exhaust gases with unburned oxygen leaves the engine across the nozzle ("7"-“9”) with producing thrust.

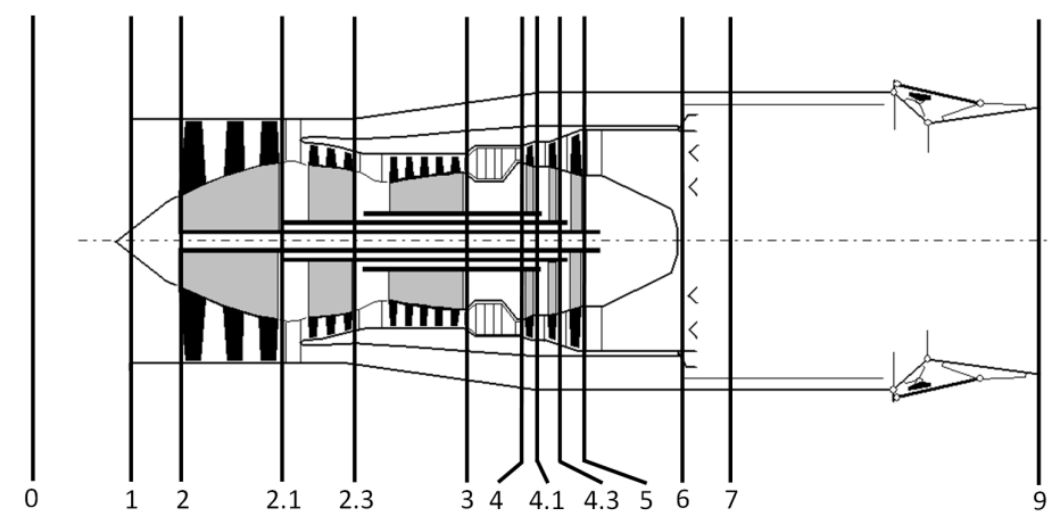

Figure 2

The layout of the mixed triple spool turbofan engine with afterburner [12]

Regarding the present investigations, real engine specifications are considered for plausibility. However, based on the available literature [9], there are known and unknown data that can be distinguished. The known parameters are the incoming air mass flow rate, pressure ratio of the compressor, turbine inlet total temperature and the length and the diameter of the engine, which are also considered as input parameters of the analyses. The unknown parameters are; efficiencies (mechanical, isentropic of compressor and turbine, burning and exhaust nozzle), losses (total pressure recovery of inlet diffuser, combustion chamber and afterburner or turbine exhaust pipe), power reduction rates of the auxiliary systems, total pressure ratio of the fan and intermediate pressure compressor, bleed air ratios for technological reasons, air income ratios due to blade cooling and total temperature at the afterburner. In order to determine these unknown parameters, constrained nonlinear optimization method is applied with the goal function to minimize the deviations between the calculated and given thrust and thrust specific fuel consumption. 
Beside the unvarying material properties, such as, specific gas constants, it is important to take the local temperature and mass fraction conditions into consideration in determining other parameters, such as, the specific heat at constant pressure and the ratio of the specific heats. These variables can be changed not only at each cross section of the engine, but also at different operational conditions belongs to different compressor pressure ratio. Equation (1) and (3) shows the expressions how they are determined as the mean value through the considered process. Equation (2) and (4) presents their standalone value at given temperature and fuel to air mass flow ratio. Iteration processes are applied if the temperature and/or fuel to air ratio is the variable of the unknown parameter so as to gain the balance between the temperature and mass fraction dependent material properties and the determined unknown thermo-dynamic parameter.

$$
\begin{aligned}
& \bar{C}_{\text {pmix }}\left(T_{i}, T_{i+1}, f\right)=\frac{1000 \sum_{j=0}^{n} \frac{a_{j}+f c_{j}}{(j+1)(f+1)}\left[\left(\frac{T_{i+1}}{1000}\right)^{j+1}-\left(\frac{T_{i}}{1000}\right)^{j+1}\right]}{T_{i+1}-T_{i}} \\
& C_{\text {pmix }}(T, f)=\sum_{j=0}^{n} \frac{a_{j}+f c_{j}}{f+1}\left(\frac{T}{1000}\right)^{j} \\
& \bar{\gamma}_{\text {mix }}=\frac{\bar{C}_{\text {pmix }}\left(T_{i}, T_{i+1}, f\right)}{\bar{C}_{\text {pmix }}\left(T_{i}, T_{i+1}, f\right)-R_{\text {mix }}} \\
& \gamma_{\text {mix }}=\frac{C_{\text {pmix }}(T, f)}{C_{\text {pmix }}(T, f)-R_{\text {mix }}}
\end{aligned}
$$

The polynomial constants for air and kerosene fuel are $a_{j}$ and $c_{j}$ according to [11]. The values of the polynomial constants for the used gases are shown in Table 1.

Table 1

The used Polynomial constants for determining the material properties of gases [11]

\begin{tabular}{|l|l|l|l|}
\hline$a_{j}$ & Value & $c_{j}$ & Value \\
\hline$a_{0}$ & 1043.797 & $c_{0}$ & 614.786 \\
\hline$a_{1}$ & -330.6087 & $c_{1}$ & 6787.993 \\
\hline$a_{2}$ & 666.7593 & $c_{2}$ & -10128.91 \\
\hline$a_{3}$ & 233.4525 & $c_{3}$ & 9375.566 \\
\hline$a_{4}$ & -1055.395 & $c_{4}$ & -4010.937 \\
\hline$a_{5}$ & 819.7499 & $c_{5}$ & 257.6096 \\
\hline$a_{6}$ & -270.54 & $c_{6}$ & 310.53 \\
\hline$a_{7}$ & 33.60668 & $c_{7}$ & -67.426468 \\
\hline
\end{tabular}

\subsection{Mathematical Model of the Triple Spool Turbojet Engine}

The used physical and mathematical approaches have been introduced in the present subchapter. 
The engine operates at sea level start condition in the present case. Total pressure recovery has been considered in the inlet diffuser of the engine. The isentropic efficiencies and pressure ratios are used at fan, intermediate and high pressure compressor for calculating the stagnation temperatures and pressures at the outlets of the units. Total pressure recovery has been considered due to the real flow modeling for determining the total pressure at the outlet of the combustion chamber. Stagnation enthalpy balance of the combustion chamber is used for determining the mass flow rate of the fuel, meanwhile the expected turbine inlet total temperature is considered. Equation (5) includes five terms as 1: stagnation enthalpy of the incoming pure air into the combustion chamber, 2: stagnation enthalpy of the fuel, 3: heat generation by the combustion, 4: stagnation enthalpy of the hot gas at the stoichiometric burning condition, which leaves the combustion chamber, 5: stagnation enthalpy of the pure air, that is found at the outlet section of the combustion chamber.

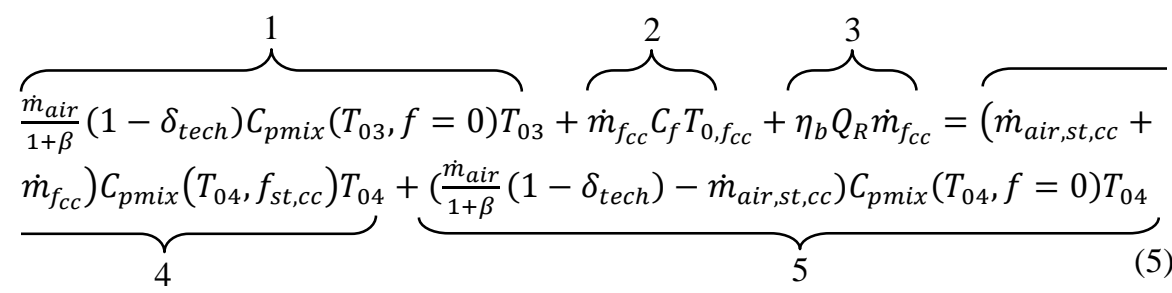

$\delta_{\text {tech }}=\dot{m}_{\text {tech }} /\left(\frac{\dot{m}_{\text {air }}}{1+\beta}\right)$ in equation (5) represents the certain amount of mass flow rate re-movements in the high pressure compressor due to the technological reason. $Q_{R}=42 \mathrm{MJ} / \mathrm{kg}$ is the lower heating value of the used fluid. $\dot{m}_{\text {air }, s t, c c}$ (see (6)) is the air mass flow rate, which is involved in the burning process at stoichiometric condition and $L_{0}=14.72 \mathrm{~kg} / \mathrm{kg}$. The definition of the fuel to air ratios in the combustion chamber is found in (7).

$\dot{m}_{\text {air }, s t, c c}=\dot{m}_{f c c} L_{0}$

$f_{c c}=\frac{\dot{m}_{f c c}(1+\beta)}{\dot{m}_{\text {air }}\left(1-\delta_{\text {tech }}\right)}, f_{s t, c c}=\frac{\dot{m}_{f c c}}{\dot{m}_{\text {air }, s t, c c}}$

Iteration cycle is applied for (5) in order to elaborate the coherent values of the fuel to air ratio and the specific heat at constant pressure.

Power equilibrium of the high pressure compressor and turbine is used to determine the total temperature at the outlet section of the high pressure turbine; $T_{04.1}$ :

$$
\begin{aligned}
& \frac{\dot{m}_{\text {air }}}{1+\beta} \bar{C}_{\text {pmix }}\left(T_{02.3}, T_{03}, f=0\right)\left(T_{03}-T_{02.3}\right)= \\
& \eta_{m} \dot{m}_{4-4.1}(1-\zeta) \bar{C}_{\text {pmix }}\left(T_{04}, T_{04.1}, f_{h p, T}\right)\left(T_{04}-T_{04.1}\right)
\end{aligned}
$$


The mas flow rate of the operational fluid in the high pressure turbine is shown in (9).

$\dot{m}_{4-4.1}=\frac{\dot{m}_{a i r}}{1+\beta}\left(1-\delta_{t e c h}\right)\left(1+f_{c c}\right)\left(1+\delta_{b c}\right)$

$\delta_{b c}$ represents the incoming air mass flow rate at high pressure turbine for blade cooling (see (10))

$\delta_{b c}=\dot{m}_{b c}(1+\beta) /\left(\dot{m}_{\text {air }}\left(1-\delta_{t e c h}\right)\left(1+f_{c c}\right)\right)$

The power balances are used also for the low and intermediate pressure units for determining the exit temperature of the low and intermediate pressure turbines. Iteration cycles are applied also for updating the specific heat at constant pressure in the turbine segments.

The total pressure recovery of the engine section 5-6 (see Figure 2.) is included in the liner location corresponds to 6-7, which approximation has negligible effect for the output of the analyses.

The by-passed and core flow are mixed at section 6. Mass flow weighted averaging procedure is applied for determining the total pressure and energy balance is considered for having the total temperature of the gas mixture.

The afterburner is located between cross section 6 . and 7. Total enthalpy balance has been considered for this segment in order to calculate the fuel mass flow rate enters into the afterburner as it is shown in (11).

$$
\begin{aligned}
& \dot{m}_{\text {air }, 6} C_{p m i x}\left(T_{06}, f=0\right) T_{06}+\dot{m}_{f, A} C_{f} T_{0, f A}+\eta_{b} Q_{R} \dot{m}_{f_{A}}+\left(\dot{m}_{\text {air }, s t, c c}+\right. \\
& \left.\dot{m}_{f_{c c}}\right) C_{p m i x}\left(T_{06}, f_{s t, c c}\right) T_{0,6}=\left(\dot{m}_{\text {air }, s t, c c}+\dot{m}_{f_{c c}}\right) C_{p m i x}\left(T_{07}, f_{s t, c c}\right) T_{07}+ \\
& \left(\dot{m}_{\text {air }, s t, A}+\dot{m}_{f_{A}}\right) C_{p m i x}\left(T_{07}, f_{s t, A}\right) T_{07}+\left(\dot{m}_{\text {air }, 6}-\dot{m}_{\text {air }, s t, A}\right) C_{p m i x}\left(T_{07}, f=\right. \\
& 0) T_{07}
\end{aligned}
$$

The left side of equation (11) shows the incoming total enthalpy into the system and the right hand side represents the leaving one. The first term of equation (11) is the stagnation enthalpy of pure air, which is available at section 6 . The second and third term is the total enthalpy of the fuel entering into afterburner and heat generated by the combustion respectively. The last term in the left hand side is the total enthalpy of the incoming hot gases, which are already burnt previously in the combustion chamber. The total enthalpy of the hot gases of the combustion chamber and the afterburner are represented by the first two terms in the right side of the (11). The last term is the total enthalpy of the pure air, which leaves the system. Iterative calculation procedure is used here also to determine the fuel to air ratio of the afterburner at stoichiometric condition $\left(f_{s t, A}=\dot{m}_{f_{A}} / \dot{m}_{a i r, s t, A}\right)$ and the specific heat at constant pressure, which are corresponds to each other by means of functional dependencies.

Expansion processes occur in the exhaust nozzle shown in Figure 3. In order to clarify whether the supposed converging nozzle is chocked or is not, temperature 
and component dependent material properties, inlet conditions of the nozzle and its isentropic efficiency are considered. If the calculated critical pressure is higher than ambient pressure, the nozzle flow is considered to be choked, which means that the exit pressure of the nozzle is equal to the critical pressure: $p_{c}$. If the ambient pressure is higher than the critical pressure, then the nozzle flow is unchocked; therefore, the exhaust pressure of the exit is equal to the ambient pressure. After having this information and determining the pressure, the temperature and the velocity at the exit of the exhaust nozzle can be calculated.
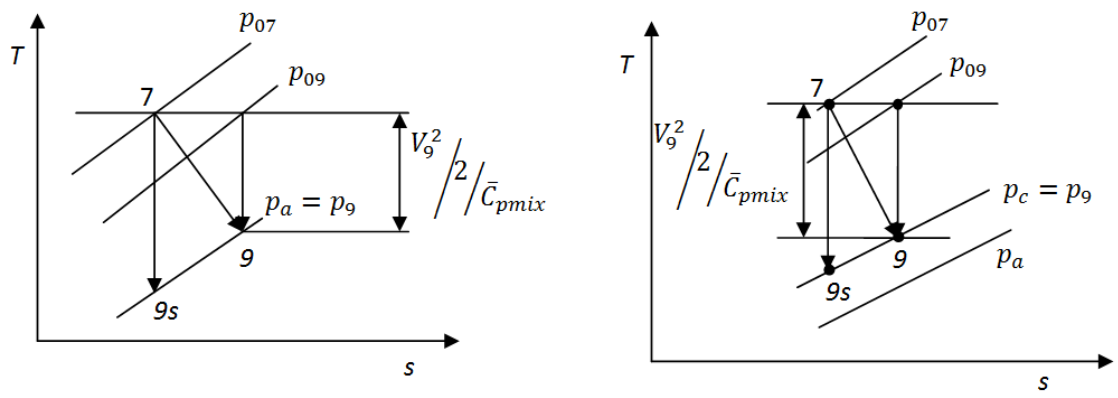

Figure 3

Nozzle flow with losses for unchoked (left side) and for choked (right side) flow conditions

A new analytical equation has been derived for determining the critical pressure at the exhaust port of the nozzle (see (18)), in which, beside the dependences of temperature variation and fuel to air ratio in the specific heat at constant pressure, the ratio of the specific heats are also considered. The critical static pressure at the outlet section of the exhaust system is coupled with the outlet static temperature; hence, iteration cycling is used for determining these variables together with the material properties in case of chocked conditions. The fuel to air ratio at the afterburner is also included in the overall fuel to air ratio:

$$
f=\left(\dot{m}_{f_{c c}}+\dot{m}_{f_{A}}\right) /\left(\dot{m}_{a i r}-\dot{m}_{t e c h}+\dot{m}_{b c}\right)
$$

The derivation of the new equation for the critical pressure at the exhaust nozzle is presented in the followings. First the total enthalpy and then total temperature at section 9 is introduced as it is shown in equation (13) and (14).

$$
\begin{aligned}
& h_{09}=h_{9}+\frac{V_{9}^{2}}{2} \Rightarrow C_{p m i x}\left(T_{09}, f\right) T_{09}=C_{p m i x}\left(T_{9}, f\right) T_{9}+\frac{V_{9}^{2}}{2} \Rightarrow T_{09}= \\
& T_{9} \frac{C_{p m i x}\left(T_{9}, f\right)}{C_{p m i x}\left(T_{09}, f\right)}+\frac{V_{9}^{2}}{2 C_{p m i x}\left(T_{09}, f\right)} \\
& T_{09}=T_{9} \frac{C_{p m i x}\left(T_{9}, f\right)}{C_{p m i x}\left(T_{09}, f\right)}+\frac{1}{C_{p m i x}\left(T_{09}, f\right)} \frac{V_{9}^{2}}{2} \frac{a_{9}^{2}}{a_{9}^{2}} \Rightarrow T_{09}= \\
& T_{9} \frac{C_{p m i x}\left(T_{9}, f\right)}{C_{p m i x}\left(T_{09}, f\right)}+\frac{1}{C_{p m i x}\left(T_{09}, f\right)} M_{9}^{2} \frac{\gamma_{\text {mix }}\left(T_{9}, f\right) R_{\text {mix }} T_{9}}{2}
\end{aligned}
$$


The critical condition corresponds to $M_{9}=1$ and $T_{9}=T_{c}$, so Equation (14) can be reformulated as follows:

$\frac{T_{09}}{T_{C}}=\frac{C_{p m i x}\left(T_{C}, f\right)}{C_{p m i x}\left(T_{09}, f\right)}+\frac{\gamma_{\text {mix }}\left(T_{\mathrm{C}}, f\right) R_{\text {mix }}}{2 C_{\text {pmix }}\left(T_{09}, f\right)}=\frac{2 C_{p m i x}\left(T_{C}, f\right)+\gamma_{\text {mix }}\left(T_{C}, f\right) R_{\text {mix }}}{2 C_{p m i x}\left(T_{09}, f\right)}$

The nozzle efficiency and the isentropic static temperature at point 9 is given by equation (16).

$\eta_{n}=\frac{C_{p m i x}\left(T_{09}, f\right) T_{09}-C_{p m i x}\left(T_{c}, f\right) T_{\mathrm{c}}}{C_{\text {pmix }}\left(T_{09}, f\right) T_{09}-C_{p m i x}\left(T_{9 s}, f\right) T_{9 \mathrm{~s}}} \Rightarrow T_{9 \mathrm{~s}}=$

$\frac{C_{p m i x}\left(T_{09}, f\right)}{C_{p m i x}\left(T_{9 S}, f\right)} T_{09}-\frac{1}{\eta_{n}} \frac{C_{p m i x}\left(T_{09}, f\right) T_{09}-C_{p m i x}\left(T_{c}, f\right) T_{\mathrm{C}}}{C_{p m i x}\left(T_{9 S}, f\right)}$

The thermodynamic process between point 7 and $9 \mathrm{~s}$ is isentropic:

$\frac{p_{C}}{p_{07}}=\left(\frac{T_{9 s}}{T_{09}}\right)^{\frac{\bar{\gamma}_{m i x}\left(T_{09, T 9 S} f\right)}{\bar{\gamma}_{m i x}\left(T_{09, T S}, f\right)^{-1}}}$

Equation (18) is appeared after inserting equation (16) and then (15) in (17) $\left(T_{09}=T_{07}\right)$.

$p_{c}=$

$p_{07}\left[\left(\frac{C_{p m i x}\left(T_{09}, f\right)}{C_{p m i x}\left(T_{9 S}, f\right)}\right)\left(1-\frac{1}{\eta_{n}}\left(1-\frac{2 C_{p m i x}\left(T_{c}, f\right)}{\left(2 C_{p m i x}\left(T_{c}, f\right)+\gamma_{\operatorname{mix}}\left(T_{c}, f\right) R_{\text {mix }}\right)}\right)\right)\right]^{\frac{\bar{\gamma}_{\operatorname{mix}}\left(T_{09}, T_{9 s}, f\right)}{\bar{\gamma}_{\operatorname{mix}}\left(T_{09}, T_{9 s}, f\right)^{-1}}}$

$\gamma_{\text {mix }}\left(T_{9}, f\right)=\frac{C_{\text {pmix }}\left(T_{9}, f\right)}{C_{p \operatorname{mix}}\left(T_{9}, f\right)-R_{\text {mix }}}$

$\bar{\gamma}_{\text {mix }}\left(T_{09}, T_{9 S}, f\right)=\frac{\bar{C}_{\text {pmix }}\left(T_{09}, T_{9 S}, f\right)}{\bar{C}_{\text {pmix }}\left(T_{09}, T_{9 s}, f\right)-R_{\text {mix }}}$

The output parameters of the analyses are the thrust and thrust specific fuel consumption, which are shown in equation (21) and (22) respectively.

$T=\left[\dot{m}_{9} V_{9}-\dot{m}_{\text {air }} V_{0}\right]+A_{9}\left(p_{9}-p_{0}\right)$

$T S F C=\frac{\dot{m}_{f c c}+\dot{m}_{f_{A}}}{T}$

The mass flow rate leaves the engine is found in equation (23).

$\dot{m}_{9}=\frac{\dot{m}_{a i r}}{1+\beta}\left[\left(1-\delta_{t e c h}\right)\left(1+f_{c c}\right)\left(1+\delta_{b c}\right)+\beta\right]$

\section{Results and Discussion}

NK-32 and NK-25 turbofan engines are used as two gas turbines to analyse and verify the simulation method. The input parameters of the two selected engines are presented in Table 2. However, as it was mentioned before, there are also several unavailable parameters, which are determined by parameter identifications. 
The missing efficiencies (mechanical, isentropic of compressor and turbine, burning and exhaust nozzle), losses (total pressure recovery factor of inlet diffuser, combustion chamber and afterburner or turbine exhaust pipe), total pressure ratio of the fan and intermediate pressure compressor, power reduction rates of the auxiliary systems, bleed air ratios for technological reasons, air income ratio due to blade cooling and total temperature of the afterburner are identified by nonlinear constrained optimization. The goal function to be minimized is the difference between the calculated and given thrust and thrust specific fuel consumption by the engine manufacturer. Tables 3, 4 and 5 show the results of the optimizations. The parameters are in the reasonable range therefore they can be accepted.

Table 2

Operational data of the NK-32 and NK-25 turbofan engines from the available specifications [9, 13]

\begin{tabular}{|c|c|c|c|c|c|}
\hline \multirow{2}{*}{$\begin{array}{l}\text { Type } \\
\text { engine }\end{array}$} & \multicolumn{5}{|c|}{ The main characteristics of the engine(at start positions) } \\
\cline { 2 - 6 } & $T_{04}(\mathrm{~K})$ & $\pi_{C}$ & $\beta$ & $\dot{m}_{\text {air }}(\mathrm{kg} / \mathrm{s})$ & $L / D(\mathrm{~m} / \mathrm{m})$ \\
\hline NK-32 & 1630 & 28.4 & 1.4 & 290 & $7.45 / 1.79$ \\
\hline NK-25 & 1600 & 26 & 1.44 & 280.9 & $7.3 / 1.442$ \\
\hline
\end{tabular}

The thrust and thrust specific fuel consumption - belongs to the parameters found in Tables 2, 3, 4 and 5 - are shown in Table 6. The relative maximal deviation between the given and the calculated data during the parameter fitting is $0.122 \%$ in case of thrust and $0.195 \%$ at the thrust specific fuel consumption. It means, together, with the plausible parameter identifications, the analysis is suitable for modeling the thermo-dynamic processes of the three spool turbojet engine.

Concerning the verification of the analyses it can be concluded, that although the both engines have the same thrust, the fuel consumption of NK-32 engine is higher due to the lower total pressure recovery factor (higher pressure loss) in the combustion chamber $\left(r_{c c}\right)$, afterburner liner $\left(r_{a l}\right)$ and intake duct $\left(r_{d}\right)$. Moreover, the NK-32 has lower mechanical, nozzle and burning efficiency beside lower fan and compressor segments isentropic efficiencies in comparing with NK-25.

Although the simulation parameters and the results are in acceptable agreement with the available data and with the expectations, more analyses will be necessary for satisfying the accuracy of the method over wider range of applications, including different engine types, operational conditions and more measured data.

Table 3

Identified efficiencies of the NK-32 and NK-25 turbofan engines

\begin{tabular}{|l|c|c|c|c|c|c|c|}
\hline & \multicolumn{7}{|c|}{ Efficiencies of the cycles } \\
\hline $\begin{array}{l}\text { Type of } \\
\text { Engine }\end{array}$ & $\eta_{m}$ & $\eta_{n}$ & $\eta_{b}$ & $\begin{array}{l}\eta_{h p, T, s} \\
\eta_{i p, T, S} \\
\eta_{l p, T, s}\end{array}$ & $\eta_{f, S}$ & $\eta_{h p, C, S}$ & $\eta_{\mathrm{ip}, \mathrm{C}, \mathrm{s}}$ \\
\hline NK-32 & 0.99 & 0.94 & 0.94 & 0.89 & 0.84 & 0.83 & 0.84 \\
\hline NK-25 & 0.992 & 0.95 & 0.97 & 0.89 & 0.86 & 0.87 & 0.87 \\
\hline
\end{tabular}


Table 4

Identified total pressure recovery factors of the 2 investigated turbofan engines

\begin{tabular}{|c|c|c|c|}
\hline & \multicolumn{3}{|c|}{$\begin{array}{l}\text { Total pressure recovery factors of comb. chamber }\left(r_{c c}\right), \\
\text { afterburner liner }\left(r_{a l}\right) \text { and intake duct }\left(r_{d}\right)\end{array}$} \\
\hline Type of Engine & $r_{c c}$ & $r_{a l}$ & $r_{d}$ \\
\hline NK-32 & 0.95 & 0.9 & 0.91 \\
\hline NK-25 & 0.96 & 0.94 & 0.94 \\
\hline
\end{tabular}

Table 5

Identified parameters of the 2 investigated turbofan engines

\begin{tabular}{|c|c|c|c|c|c|c|}
\hline & \multicolumn{6}{|l}{$\begin{array}{l}\text { Total pressure ratio of the fan }\left(\pi_{f}\right) \text { and intermediate pressure } \\
\text { compressor }\left(\pi_{i p, c}\right) \text {, bleed air ratio for technological reasons }\left(\delta_{\text {tech }}\right), \\
\text { air income ratio due to blade cooling }\left(\delta_{b c}\right) \text {, power reduction rates } \\
\text { for the auxiliary systems }(\xi) \text { and total temperature of the afterburner } \\
\left(T_{07}\right)\end{array}$} \\
\hline Type of Engine & $\pi_{f}$ & $\pi_{i p, C}$ & $\delta_{\text {tech }}$ & $\delta_{b c}$ & $\xi$ & $T_{07}(\mathrm{~K})$ \\
\hline NK-32 & 2 & 3 & 0.16 & 0.116 & 0.005 & 1800 \\
\hline NK-25 & 2.19 & 3 & 0.18 & 0.0952 & 0.005 & 1780 \\
\hline
\end{tabular}

Table 6

Comparisons of the available data with the outputs of the parameter fitting

\begin{tabular}{|c|c|c|c|c|}
\hline $\begin{array}{l}\text { Type } \\
\text { Engine }\end{array}$ & \multicolumn{2}{|l|}{$\begin{array}{l}\text { Available data } \\
\text { (start position })\end{array}$} & \multicolumn{2}{l}{$\begin{array}{l}\text { Outputs of the parameter fitting } \\
\text { (start position })\end{array}$} \\
\hline & $T(\mathrm{kN})$ & $T S F C(\mathrm{~kg} /(\mathrm{kN} \mathrm{h}))$ & $T(\mathrm{kN})$ & $T S F C(\mathrm{~kg} /(\mathrm{kN} \mathrm{h}))$ \\
\hline NK-32 & 245 & - & 245.1 & 229.4 \\
\hline NK-25 & 245 & 205.3 & 245.3 & 205.7 \\
\hline
\end{tabular}

The thermo-dynamic cycles of the engine processes are found in Figure 4. The red curve-sections (or lighter and thinner in grayscale) represent the constant pressures in the T-s diagrams (total from points 0-7 and static at 9). The processes between the engine states denoted, by numbers, are plotted by thicker lines. This visualization effect is the reason of the constant pressure line goes below the process line in case of pressure decrement just after section " 3 ".

The analyses of the NK-32 and NK-25 engines are also completed by using the conventional equation (24) for determining the critical pressure at the exit of the converging nozzle in case of the same thermo-dynamic conditions.

$p_{c}=p_{07}\left(1-\frac{1}{\eta_{n}}\left(\frac{\gamma_{g a s}-1}{\gamma_{g a s}+1}\right)\right)^{\frac{\gamma_{g a s}}{\gamma_{g a s}-1}}$

The effect of the fuel to air ratio and temperature are not considered in the ratio of specific heat in (24), the flow is considered to be pure gas with $\gamma_{\text {gas }}=1.33$. The results of the calculations are shown in Table 7 . The average deviation between the resulted and the available thrust and thrust specific fuel consumption is 
$0.119 \%$ by using the new equation (18) and $3.13 \%$ at the conventional equation (24).
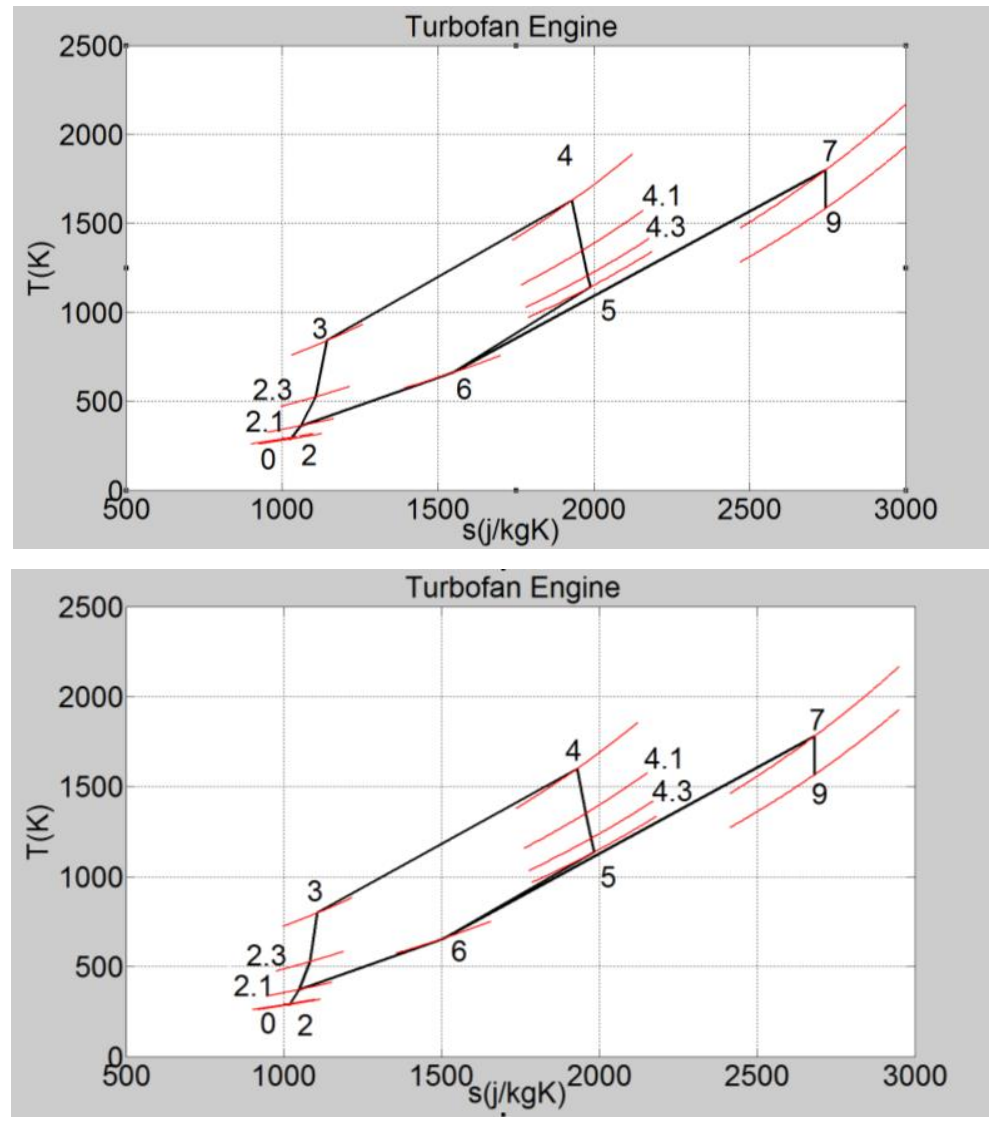

Figure 4

Thermo-dynamic cycle of the NK-32 (top) and NK-25 (bottom) turbofan engine with afterburner (the red curve sections (the lighter thinner ones in grayscale) in the T-s diagrams are the constant pressure curves (total from 0-7 and static at 9) belong to the shown numbers of the engine cross sections)

Table 7

Comparison the effect of the conventional and the new equation on the thrust and the thrust specific fuel consumption in case of NK-32 and NK-25 turbofan engines

\begin{tabular}{|c|c|c|c|c|}
\hline $\begin{array}{c}\text { Type of } \\
\text { Engine }\end{array}$ & \multicolumn{2}{|c|}{ Relative difference (new equation) } & \multicolumn{2}{|c|}{$\begin{array}{c}\text { Relative difference (conventional } \\
\text { equation) }\end{array}$} \\
\hline & $T(\mathrm{kN})$ & $T S F C(\mathrm{~kg} /(\mathrm{kNh}))$ & $T(\mathrm{kN})$ & $T S F C(\mathrm{~kg} /(\mathrm{kNh}))$ \\
\hline NK-32 & $0.04 \%$ & - & $3.27 \%$ & - \\
\hline NK-25 & $0.122 \%$ & $0.195 \%$ & $2.85 \%$ & $3.27 \%$ \\
\hline
\end{tabular}




\section{Conclusions}

A thermo-dynamic model has been developed and implemented in the MATLAB environment, for determining the main characteristics of the triple spool turbojet engines with afterburner. The mass, energy balance and the real thermo-dynamic process equations are used in the concentrated parameter distributions type model, in which the mechanical, isentropic and burning efficiencies, pressure losses and the bleed air ratio, for technological reasons, air ratio for blade cooling, fan and intermediate compressor pressure ratios, the afterburner temperature and power reduction rate of the auxiliary systems are considered. Nonlinear constraint optimization is applied for determining the mentioned data by means of fitting the calculated the thrust and thrust specific fuel consumption - if it was available - to the known parameters, which are given in the specification. Ambient conditions, incoming air mass flow rate, pressure ratio of the compressor, turbine inlet total temperature, the length and diameter of the engine are the input parameters of the analyses. The material properties, such as, specific heat and the ratio of specific heat depends on the temperature and component mass fraction and so they are determined by iteration cycles, in case of functional dependencies.

The calculated thrust and thrust specific fuel consumption of the NK-32 and NK25 engines by parameter fitting and the available data are compared with each other at start conditions. The results show that the relative maximum deviation between the given and the calculated parameters is $0.122 \%$ in case of thrust and $0.195 \%$ at the thrust specific fuel consumption, meanwhile the identified parameters are within the plausible range.

A new analytical equation has been derived for determining the critical pressure at the exit of the converging nozzle, in which, beside, the dependences of temperature variation and fuel to air ratio in the specific heat at constant pressure, the ratio of the specific heats is also included. The new equation provides $3.01 \%$ (delta) improvements with respect to the conventional formula, for the case of averaged deviation between the resulted and the available thrust and thrust specific fuel consumption for the investigated engine types at the same condition and parameters.

\section{References}

[1] Bréda R., Lazar T., Andoga R. and Madarász L.: Robust Controller in the Structure of Lateral Control of Maneuvering Aircraft, Acta Polytechnica Hungarica, pp. 101-124, Vol. 10, No. 5, 2013

[2] Gáti B., and Drouin A.: Open Source Autopilot for Academic Research The Paparazzi System, Proceedings of the American Control Conference, Washington DC: AACC International, 1-6. ISBN: 978-1-4799-0175-3, 2013

[3] Voskuilj M., Rohács D., Rohács J. and Schousttra R. J.: Preliminary Evaluation of the Environmental Impact related to Aircraft Take-off and 
Landings supported with Ground Based (MAGLEV) Power, Proceedings of the $4^{\text {th }}$ Annual International Air Transport and Operations Symposium (ATOS), Toulouse, French, 10.07.2013-12.07. 2013

[4] Beneda K. and Rohács J.: Dynamic Model of Variable Inducer Shroud Bleed for Centrifugal Compressor Surge Suppression, International Review of Aerospace Engineering 6, 163-173, Paper online, 2013

[5] Bera J. and Pokorádi L.: Monte-Carlo Simulation of Helicopter Noise, Acta Polytechnica Hungarica, 12:(2) pp. 21-32, 2015

[6] Andoga R., Főző L., Madarász L. and Karol T.: A Digital Diagnostic System for a Small Turbojet Engine, Acta Polytechnica Hungarica, pp. 4558, Vol. 10, No. 4, 2013

[7] Abhijit G.: Optimum Fan Pressure Ratio for Bypass Engines with Separate or Mixed Exhaust Streams, Propulsion and power J, Vol. 17, No. 5, 2001, $1117-1122$

[8] Silva V. V. R., Khatib W. and Fleming P. J.: Performance Optimization of Gas Turbine Engine, Artificial Intelligence, Vol. 18, No. 5,575-583, Aug. 2005

[9] Кулагин В. В.: Теория расчёт и проектирование авиационных двигателей и энергетических установок. Книга 1, 2, Moscow, 2005

[10] http://www.uk-odk.ru/img/content/products/NK-32_big.png, 15.06.2014

[11] Sánta I.: Gázturbinás Repülőgép Hajtómüvek, BME Repülőgépek és Hajók Tanszék, tanszéki jegyzet (Lecture Notes on Jet Engines at BME University), 2008

[12] Kurzke J.: http://www.gasturb.de/, 03.03.2014

[13] https://en.wikipedia.org/wiki/Kuznetsov_NK-32, 01.05.2016

[14] https://en.wikipedia.org/wiki/Kuznetsov_NK-25, 01.05.2016 